\title{
Bio-inspired Nano-photodiode for Low Light, High Resolution and Crosstalk-free CMOS Image Sensing
}

\author{
Fayçal Saffih \\ Physical Science and Engineering, King Abdullah University \\ for Science and Technology \\ 4700 King Abdullah University of Science and Technology \\ Thuwal 23955-6900, KSA \\ Email: faycal.saffih@kaust.edu.sa
}

Nathaniel N. Fitzpatrick, Mohammad A. Mohammad and Stephane Evoy

Department of Electrical and Computer Engineering, University of Alberta, Edmonton, AB T6G 2V4, Canada \{nathan.fitzpatrick, mam20, evoy\}@ece.ualberta.ca Bo Cui

Electrical and Computer Engineering Department, University of Waterloo, 200 University Avenue West Waterloo, ON, Canada, N2L3G1 bcui@uwaterloo.ca

\begin{abstract}
Previous attempts have been devoted to mimic biological vision intelligence at the architectural system level. In this paper, a novel imitation of biological visual system intelligence is suggested, at the device level with the introduction of novel photodiode morphology. The proposed bio-inspired nanorod photodiode puts the depletion region length on the path of the incident photon instead of on its width, as the case is with the planar photodiodes. The depletion region has a revolving volume to increase the photodiode responsivity, and thus its photosensitivity. In addition, it can virtually boost the pixel fill factor (FF) above the $100 \%$ classical limit due to decoupling of its vertical sensing area from its limited planar circuitry area. Furthermore, the suggested nanorod photodiode photosensitivity is analytically proven to be higher than that of the planar photodiode. We also show semi-empirically that the responsivity of the suggested device varies linearly with its height; this important feature has been confirmed using Sentaurus simulation. The proposed nano-photorod is believed to meet the increasingly stringent High-Resolution-Low-Light (HRLL) detection requirements of the camera-phone and biomedical imaging markets.
\end{abstract}

\section{INTRODUCTION}

Electronic imaging technologies have tremendously progressed in the last three decades for the maturing CoupledCharge Devices (CCD) technology and in the last two decades for the emerging Complementary-Metal-OxideSemiconductor Active Pixel Sensor imaging (CMOS-APS) technology [1]-[4]. Enhancing the resolution of CMOS image sensors is always an important factor of this imaging technology evolution [4]. However, the progress is likely to face a great obstacle for CMOS APS as the Signal-to-Noise Ratio (SNR) and Dynamic Range (DR) go inversely with the pixel pitch [4]. These constraints are, however, not faced in CCD's due to minimal dielectric stack layers over the pixels and the larger full well capacity of the CCD's MOS structure. These technological constraints have led to hybridizing CCD's image sensing elements with CMOS low power, mixed signal processing and faster readout to achieve sub-micron pixel resolution [5]. However, combining CCD within CMOS technology does not import the former high charge transfer, lower dark current and surface traps into the latter single polysilicon limitation and surface traps [6].

At the technological level, CMOS imaging is based on the vertical PN junction adopted from the planar standard CMOS technology. The small depletion region and its short intersection with incident photons, which limits the photosignal, inhibit smaller photodiode design and higher resolution CMOS imagers. The matter becomes worse in low light conditions where fewer photons impinge on the active area. To tackle these device constraints, photo-generation of charges has to take place in a 3D surface area instead of the current $2 \mathrm{D}$ planar photodiodes. In fact, biological smart visual systems use non-planar photocells of conic and cylindrical morphologies [7] to maximize vision resolution without compromising low light vision. Figure 1 shows the anatomy of a human retina and its cross section. Biological photocells sense the light along their elongations; this enhances their light sensitivity and relaxes their footprint area constraints.

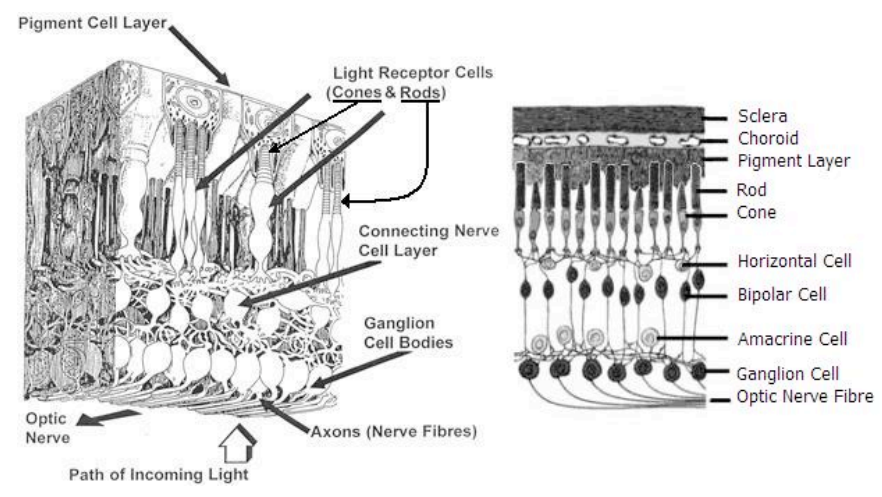

Figure $1.3 \mathrm{D}$ view and a cross section of human retina ${ }^{1}$.

${ }^{1} \mathrm{http}: / /$ www.detectingdesign.com/humaneye.html 
In this regard, we suggest a nanophotonic rod (NPR) structure [8] to replace the planar photodiode. It is a nanophotonic pillar of n-type (cathode) semiconductor covered by a p-type (anode) semiconductor as shown in Figure 2. The suggested bio-mimetic photodiode uses its depletion length instead of width in the pathway of the impinging light to enhance its sensitivity and reduce its footprint area constraint. Previous attempts using nanowires for photo-detection have been suggested but not in the biomimicking context [9].

The importance of NPR based pixel for electronic imaging relies on the following characteristics:

1. Avoiding the HRLL stringent requirements microlenses are used in the planar photodiodes to enhance the effective pixel fill factor especially, which becomes even harder with increasing resolution requirements. NPR structure alleviates this requisite by eliminating the need for microlenses altogether due to its very high fill factor as will be demonstrated in the next section.
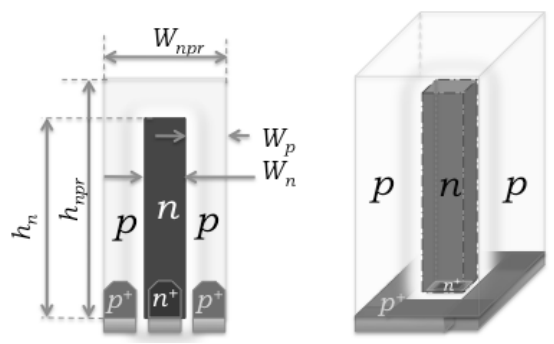

Figure 2. The cross-section and $3 \mathrm{D}$ view of NPR structure

2. Boosting the current imaging resolution to higher levels, thus benefitting more applications in biomedical imaging and communication systems.

The remainder of the paper is organized as follows: in section I, we present the photosensitivity analysis and show how the geometry of the photodiode impacts it. Next, a semiempirical analysis of the NPR based photodiode sensitivity and responsivity will be presented. The resulting dependence of the NPR photo-detection sensitivity on its height is proven and supporting examples from biological photocells are discussed. Finally, and before concluding the paper, we show some simulation results carried out using Sentaurus tools from Synopsys.

\section{SENSITIVITY ANALYSIS}

One of the most important features characterizing active pixel sensors is their photosensitivity that relates two important factors, the conversion gain $(C G)$ and the quantum efficiency $(Q E)$ as derived from the following:

$$
\text { Vout }=\#\left(e_{p h}^{-}\right) \times C G
$$

$\#\left(e_{p h}^{-}\right)$is the number of the generated photo-electrons. However, $C G=q / C_{P D}^{\text {int }}$, where $q$ is the electron charge and $C_{P D}^{\text {int }}$ is the photodiode capacitance at the integration node which can be written as: $C_{P D}^{\text {int }}=A^{\text {int }} \times C_{P D}^{A}$, where $A^{\text {int }}$ and $C_{P D}^{A}$ are the area the integration node areal capacitance respectively. Therefore,

$$
\begin{aligned}
& V o u t=Q E \times(\# p h) \times C G \\
& \text { and } Q E=\left(\# e_{p h}^{-}\right) /(\# p h)
\end{aligned}
$$

$(\# p h)$ is the number of interacting photons which can be calculated as:

$$
(\# p h)=\frac{\Phi_{i n c} \times A^{g e n} \times T^{\mathrm{int}}}{h v}
$$

$A^{\text {gen }}$ is the generating area where the electron-hole pairs are photo-generated, $T^{\text {int }}$ is the integration time, $h$ is the Plank constant, $v$ is the photon frequency and $\Phi_{i n c}$ is the incident light interacting flux.

Therefore,

$$
\text { Vout }=Q E \times \frac{\Phi_{i n c} \times A^{g e n} \times T^{\mathrm{int}}}{h v} \times C G
$$

By definition, the sensitivity is the derivation of the output voltage with respect to light intensity and integration time. This derivation is taken in the linear region of the photon transfer curve of the pixel and this derivation can be replaced by the variation. Thus, we get the following equation.

$$
S=\frac{V_{\text {out }}}{\Phi_{\text {inc }} \times T^{\mathrm{int}}}=\frac{Q E \times A^{g e n}}{h v} \times C G
$$

On the other hand, the responsivity is defined as:

$$
R_{e}=Q E \times \frac{q}{h v}
$$

Therefore, the sensitivity can be derived from equations (6) and (7) as the following:

$$
S=\frac{R_{e}}{q} \times A^{g e n} \times C G=R_{e} \times \frac{A^{g e n}}{A^{\mathrm{int}}} \times \frac{1}{C_{P D}^{A}}
$$

Two cases, which differentiate two major types of APS sensors, follow from the above equation. When $A^{\text {gen }} \neq A^{\text {int }}$, then the integration node is separated from the generation node; therefore, minimizing $A^{\text {int }}$ relative to $A^{\text {gen }}$ will enhance the sensitivity. This is the case of the $4 \mathrm{~T}$ photogate APS sensor [11], where the floating diffusion, which plays the role of the integration node, is made as small the technology allows, while the photodiode is basically a large CCD MOS structure. In the other case, when $A^{\text {gen }}=A^{\text {int }}$, then the sensitivity $S$ will be independent explicitly of the photodiode geometry and is only dependent on the device's physical characteristics. This case corresponds to the three-transistor (3T) APS configuration.

The geometry of the 3T APS photodiode might influence its sensitivity through the responsivity $R_{e}$, which depends on the depletion region width, besides other parameters. This 
width is the path length of the trajectory of the photon passing by the depletion region.

The suggested NPR photodiode enhances its sensitivity as explained in the following:

1. The anode can be fully depleted by lowering its doping concentration, thus expanding the depletion region width to the whole anode thickness. Consequently, the photodiode areal capacitance $C_{P D}^{A}$ is reduced and its responsivity $R_{e}$ enhanced

2. By having the depletion region perpendicular to the photon pathway, the electron-hole pair EHP recombination in the depletion region is diminished. This is due to the fact that as soon as EHP is created, electron and hole charges are separated by the junction internal electric field. Therefore, the number of collected charges increases, thus boosting the photodiode responsivity $R_{e}$.

3. The enhancement in the previous factor will be further improved because the depletion region volume is higher, compared to a similar photodiode, thanks to its high aspect ratio. The depletion region volume can be increased by widening the thickness of the anode, which in addition reduces the areal capacitance $C_{P D}^{A}$ as discussed in the first factor. Thus, increasing the anode thickness has double enhancing effect on the overall responsivity. The drawback might be the increase of transit time of photo-charges to reach their collection nodes.

4. Finally, in the case of full depletion, the effect of diffused dark current from the silicon dead area that is absent from the NPR photodiode structure is minimal. The dark current can also be generated by the defects located in the depletion region. However, it is thought that their contribution may be minimal due to the built-in field.

The absence of a dead zone in the NPR, or in between NPR-photodiode-based pixels cancels out any photoelectrical crosstalk between pixels. This minimal crosstalk property is absent in current CMOS imaging technology based on the planar photodiode. This is due to the relatively large volume of dead semiconductor area from which photo-thermal currents diffuse randomly to the adjacent pixels, thus increasing crosstalk.

All previously mentioned arguments of sensitivity and crosstalk enhancements are based on the physical aspects of the PN junction as discussed in the literature [12]. The results of the above analysis can also be verified using device simulators such as Sentaurus from Synopsys, which is currently underway.

\section{SEMI-EMPIRICAL SENSITIVITY ANALYSIS}

To strengthen previous section predictions, a widely accepted semi-empirical formula for the pixel photosensitivity will be adopted [13] and re-written as:

$$
S=\frac{C_{1} A+C_{2}(f(A)+P d)((T-A) / T)\left(1-L / L_{d i f f}\right)}{\left(C_{A} A+C_{P} P\right) / q}(9)
$$

$C_{1}$ and $C_{2}$ are empirical fitting parameters, $A$ and $T$ are the pixel active (i.e. $A=N P R \_b a s e$ ) and total areas, respectively. $L$ and $L_{\text {diff }}$ are the maximum and diffusion lengths of the photogenerated carriers respectively, and $C_{A}$ and $C_{P}$ are respectively the photodiode areal and peripheral capacitances. Finally, $P$ and $d$ are the photodiode perimeter and junction depth respectively, and $f(A)$ is a function of the area accounting for the planar photodiode bottom area photo-charge contribution. The second term of the numerator considers the peripheral diffusion current contribution (lateral and bottom contributions). Assuming a negligible NPR photodiode footprint area, i.e. $N P R$ base, and a minimal areal contribution $f(A) \sim 0$, the NPR sensitivity can be derived from equation (9) as:

$S \cong \frac{C_{2} P d\left(1-L / L_{\text {diff }}\right)}{\left(C_{P} P\right) / q}=q \frac{C_{2} h\left(1-L / L_{\text {diff }}\right)}{C_{P}}$

, which can be further simplified, knowing that the NPR diameter is much smaller than $L_{\text {diff }}$, to the following equation:

$$
S \cong \frac{q C_{2}}{C_{P}} h
$$

Equation (11) is a non-intuitive as it relates mathematically the NPR photodiode sensitivity $S$, on one hand, and its geometrical $(h)$ and electrical properties $\left(C_{2}\right.$ and $\left.C_{P}\right)$ on the other. The parameters controlling the NPR photodiode sensitivity are its height $h$ and its peripheral capacitance $C_{P}$. Therefore, enhancing NPR photodiode sensitivity can be achieved either by minimizing $C_{P}$ or by enlarging NPR height $h$ or both. The peripheral capacitance $C_{P}$ can be reduced by fully depleting the NPR photodiode with a relatively thicker anode. This explains the NPR photodiode sensitivity enhancement factors discussed in the previous section. The technique of enlarging NPR photodiode height $h$, however, is biologically implemented by light-controlled height-extension in bio-photocells. This phenomenon has been observed in the biological photocells of mammalians such Bullfrogs. They adapt the length of their photocells (cones): elongating them in dim lights and shortening them in bright lights [14]. A similar phenomenon is present in non-mammalian species such as Haplochromis burtoni (fish) [15].

\section{FABRICATION AND SIMULATION ANALYSIS}

In this section some simulation results carried out using Sentaurus Work Bench are presented. The NPR structures have been fabricated using MEMS compatible processes starting from N-doped wafer. First, we used Electron-Beam Lithography (EBL) to pattern an array of disks and the lift-off masking technique to mask them with Chromium to make various nanopillars in terms of diameter and pitch. The height is realized by etching the non-masked area using InductiveCoupling Plasma Reactive Ion Etching (ICPRIE). Next, the doping phase is realized by ion-implanting the surface of the nanorods with Boron to create P-doped anode layer. All the previously mentioned processes have low temperature thus are CMOS compatible for future integration. Finally, contact areas, to NPR cathode and anode layers, were created by 
heavily implanting these areas to make them ohmic. This will allow probing for NPR structure characterization.

Many sample arrays of NPR rods have been fabricated along with their probing contacts as depicted by Figure 3. The device is currently under test for its electro-optical characterization.

Simulation carried out using Sentaurus work bench, a Synopsys TCAD tool, was realized on a single $0.7 \mathrm{um}$-wide NPR photodiode to characterize its photo-electrical response versus various heights at a light wave length of $0.5 \mathrm{um}$ and intensity of $1 \mathrm{~W} / \mathrm{cm}^{2}$. Figure 4 shows the dependence of the NPR photoresponsivity versus its height. The curve shows a linear trend of the NPR responsivity versus its height for size below $0.7 \mathrm{um}$ and reaches saturation there after. This could be related to the fact that most of the light power is absorbed within $\operatorname{lum}\left(\alpha=1.82 \mathrm{um}^{-1}\right)$ of the absorption surface.

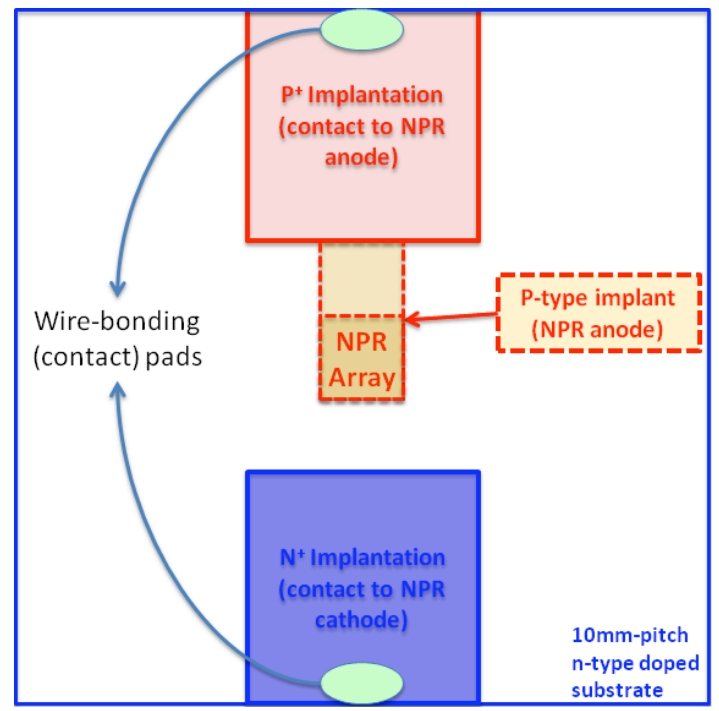

Figure 3. Schematic of the NPR test implementation.

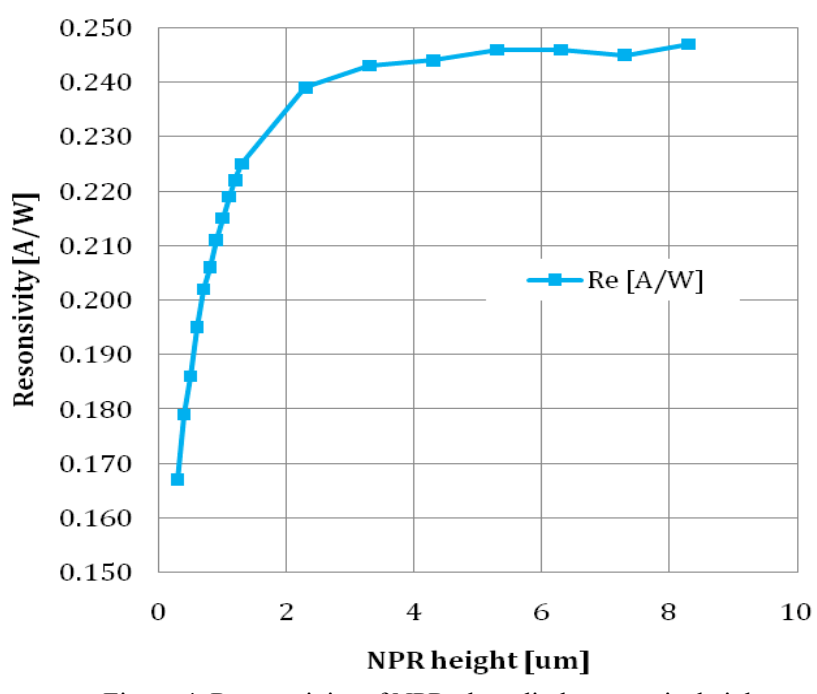

Figure 4. Responsivity of NPR photodiode versus its height

Figure 5 show an SEM picture of the fabricated NPR photodiode to be soon tested.

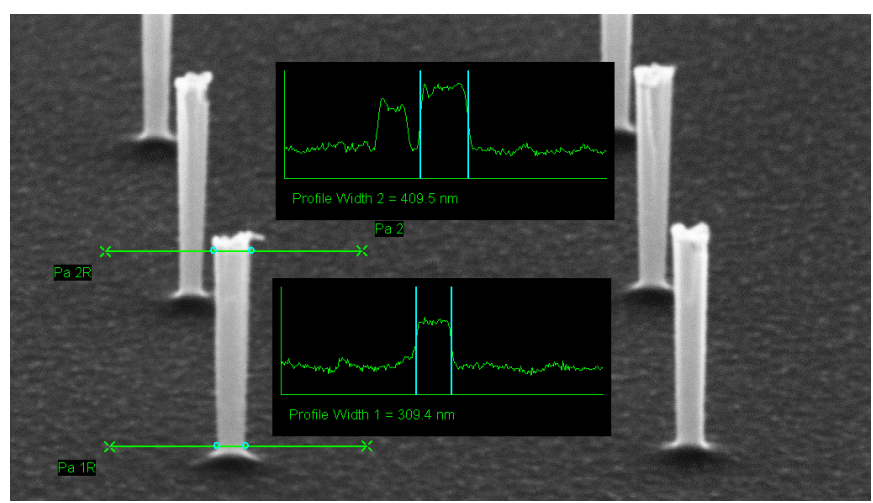

Figure 5. SEM of a fabricated NPR using liftoff and deep ICPRIE

\section{CONCLUSION}

Novel photodiode structure devices have been suggested, and their sensitivity analyzed. The suggested non-planar photodiode is proven to be the solution for the ever-increasing demand of image sensing resolution with enhanced image quality performance. The implementation aspects and the integrability of the suggested device into the mainstream CMOS technology are not expected to be a challenge and are currently being explored.

\section{REFERENCES}

[1] Fayçal Saffih, Richard Hornsey, "Foveated Dynamic Range of the Pyramidal CMOS Image Sensors", IEEE Transactions on Electron Devices, Vol. 54, $\mathrm{N}^{\circ}$ 12, December 2007, pp. 3422-3425.

[2] Fayçal Saffih, Richard Hornsey, "Pyramidal CMOS Imager FPN noise Reduction through Human Visual System Perception" IEEE Transactions on Circuits and Systems for Video Technology, Vol. 17, Issue: 7, July 2007, pp. 924-930.

[3] Fossum ER., "Active pixel sensors versus CCDs". IEEE workshop on CCDs \& AIS, 1993.

[4] Albert J.P. Theuwissen, "CMOS image sensors: State-of-the-art ", Solid-State Electronics, Vol. 52, Issue 9, Sept. 2008, Pages 1401-1406

[5] K. Fife, A. El Gamal, and H. Wong, "A 0.5 um pixel frame-transfer CCD image sensor in $110 \mathrm{~nm}$ CMOS," in Proc. IEDM, Dec. 2007, pp.1003-1006.

[6] K. Fife, A. El Gamal, and H. Wong, "A Multi-Aperture Image Sensor With 0.7 um Pixels in 110nm CMOS", IEEE Journal of Solid-State Circuits, Vol. 43, Issue 12, pp. 2990-3005, Dec. 2008.

[7] B. A. Wandell, "Foundation of vision", Ed. Sunderland, 1995.

[8] U.S. Patent Pending Application No. 61/299,829

[9] C. Soci et all., "ZnO Nanowire UV Photodetectors with High Internal Gain" Nano Letters, 2007, 7 (4), pp 1003-1009

[10] B. Dierickx, G. Meynants, D. Scheffer, Near $100 \%$ fill factor CMOS active pixels, Extended programme of the 1997 IEEE CCD \& Advanced Image Sensors Workshop, Belgium.

[11] Sunetra Mendis, Sabrina E. Kemeny, and Eric R. Fossum, "CMOS Active Pixel Image Sensor" IEEE Trans. Elec.Dev. Vol.41, No. 3, March 1994.

[12] Sze, S. M. Physics of Semiconductor Devices, Wiley-Int., 1981.

[13] Beatriz Blanco-Filgueira et al."Modeling and simulation of CMOS APS", Proceedings of the 2009 Spamish Conference on Electron Devices, Feb 11-13, 2009, Santiago de Compostela, Spain, pp. 120-123

[14] Roy H. Steinberg, "Scanning Electron Microscope of the Bullfrog's Retina and Pigment Epithelium", Cell and Tissue Research Journal, Springer-Verlag, Vol. 143, No 4, pp.451-463, December 1973

[15] Ilsabe Pietzsch-Rohrschneider, "Scanning Electron Microscopy of Photoreceptor Cellsin the Light- and Dark-Adapted Retinaof Haplochromis burtoni (Cichlidae, Teleostei)", Cell and Tissue Research Journal, Springer-Verlag, Vol. 175, No 1, pp. 123-130, Nov. 197 